\title{
Improved production of fatty acid ethyl esters in Saccharomyces cerevisiae through up-regulation of the ethanol degradation pathway and expression of the heterologous phosphoketolase pathway
}

Bouke Wim de Jong ${ }^{1}$, Shuobo Shi ${ }^{2}$, Verena Siewers ${ }^{1}$ and Jens Nielsen ${ }^{1 *}$

\begin{abstract}
Background: Due to an increasing demand of transportation fuels, a lower availability of cheap crude oil and a lack of sustainability of fossil fuels, a gradual shift from petroleum based fuels towards alternative and renewable fuel resources will be required in the near future. Fatty acid ethyl esters (FAEEs) have properties similar to current crude diesel and could therefore form an important contribution to the development of sustainable transportation fuels in future. It is important to develop novel cell factories for efficient production of FAEEs and their precursors.

Results: Here, a Saccharomyces cerevisiae cell factory expressing a heterologous wax ester synthase (ws2) from Marinobacter hydrocarbonoclasticus was used to produce FAEEs from ethanol and acyl-coenzyme A (acyl-CoA). The production of acyl-CoA requires large amounts of NADPH and acetyl-CoA. Therefore, two metabolic engineering strategies for improved provision of NADPH and acetyl-CoA were evaluated. First, the ethanol degradation pathway was employed to re-channel carbon flow towards the synthesis of acetyl-CoA. Therefore, ADH2 and ALD6 encoding, respectively, alcohol dehydrogenase and acetaldehyde dehydrogenase were overexpressed together with the heterologous gene aCS ${ }_{S E}{ }^{\text {G64PP }}$ encoding acetyl-COA synthetase. The Co-overexpression of ADH2, ALD6 and aCS ${ }_{S E}^{\text {L641P }}$ with ws2 resulted in $408 \pm 270 \mu \mathrm{g} \mathrm{FAEE} \mathrm{gCDW}^{-1}$, a 3-fold improvement. Secondly, for the expression of the PHK pathway two genes, $x p k A$ and ack, both descending from Aspergillus nidulans, were co-expressed together with ws2 to catalyze, respectively, the conversion of xylulose-5-phosphate to acetyl phosphate and glyceraldehyde3-phosphate and acetyl phosphate to acetate. Alternatively, ack was substituted with pta from Bacillus subtilis, encoding phosphotransacetylase for the conversion of acetyl phosphate to acetyl-CoA. Both PHK pathways were additionally expressed in a strain with multiple chromosomally integrated ws 2 gene, which resulted in respectively $5100 \pm 509$ and $4670 \pm 379 \mu \mathrm{g} \mathrm{FAEE} \mathrm{gCDW}^{-1}$, an up to 1.7 -fold improvement.

Conclusion: Two different strategies for engineering of the central carbon metabolism for efficient provision of acetyl-COA and NADPH required for fatty acid biosynthesis and hence FAEE production were evaluated and it was found that both the ethanol degradation pathway as well as the phosphoketolase pathway improve the yield of FAEEs.
\end{abstract}

Keywords: Saccharomyces cerevisiae, Fatty acid ethyl ester (FAEE), Biodiesel, Metabolic engineering

\footnotetext{
*Correspondence: nielsenj@chalmers.se

'Department of Chemical and Biological Engineering, Chalmers University of

Technology, Kemivägen 10, Göteborg SE-412 96, Sweden

Full list of author information is available at the end of the article
} 


\section{Background}

There is much interest in developing novel cell factories for production of advanced biofuels that can be used as diesel and bunker oil [1], and here we describe production of fatty acid ethyl esters (FAEEs) by yeast. The principle of FAEE production has been demonstrated in Escherichia coli as well as in Saccharomyces cerevisiae where FAEEs were formed by a transesterification reaction between ethanol and fatty acyl-CoA, which was catalyzed by a heterologous wax ester synthase/acyl-CoA:diacylglycerol acyltransferase (WS/DGAT) [2,3]. The enzyme activities of several WS/DGATs have been analyzed [4]. Five different wax ester synthase genes from different origins were expressed in $S$. cerevisiae and a strain expressing a wax ester synthase (ws2) from Marinobacter hydrocarbonoclasticus DSM 8798 showed the highest production of FAEEs $[4,5]$.

While oleaginous organisms such as Botryococcus braunii, Gordonia sp., Humicola lanuginose or Lipomyces starkeyi were reported to accumulate lipids up to $65-75 \%$ of their cell dry weight, the yeast $S$. cerevisiae is a natural ethanol producer and not an oleaginous organism. There is therefore a demand for engineering strategies targeting the synthesis of acyl-CoA, one of the precursors for FAEE production [6-10].

The precursor for fatty acid biosynthesis is acetyl coenzyme A (acetyl-CoA), which plays a central role in several cellular pathways and compartments, and is involved in regulatory mechanisms $[11,12]$. In $S$. cerevisiae, it is produced in the cytosol, the mitochondria and the peroxisomes, but is not able to cross the organelle membranes. Acetyl-CoA also functions as a gateway for metabolic routes to many other biotechnologically valuable compounds, the pathways of which are mostly targeted to the cytosol and therefore there consists an interest to increase the production of acetyl-CoA in the cytosol [12,13].

During growth of $S$. cerevisiae on high concentrations of glucose, most glycolytic carbon is directed towards the production of ethanol from acetaldehyde which is known as the Crabtree effect $[14,15]$. To reduce the amount of ethanol and increase the amount of cytosolic acetyl-CoA, a metabolic engineering strategy described by Chen et al. [13] was applied. Alcohol dehydrogenase 2 (Adh2) catalyzes the conversion of ethanol to acetaldehyde in $S$. cerevisiae. For the formation of cytosolic acetyl-CoA, acetaldehyde is converted to acetate, which is catalyzed by acetaldehyde dehydrogenase encoded by endogenous $A L D 1$ - ALD7 and subsequently to acetylCoA catalyzed by acetyl-CoA synthetase (encoded by $A C S 1$ and $A C S 2)[12,16]$. In this approach, endogenous ALD6 and a heterologous ACS variant from Salmonella enterica encoded by $a c S_{S E}{ }^{L 641 P}$ were overexpressed. The ACS variant $\operatorname{acs}_{S E}{ }^{L 641 P}$ carries an amino acid substitution that prevents the enzyme from being inactivated by acetylation $[17,18]$. The metabolic pathway is shown in Figure 1A.

For the synthesis of acyl-CoA, acetyl-CoA forms the primer which is extended by addition of $\mathrm{C} 2$ units derived from malonyl-CoA. For each new addition of a $\mathrm{C} 2$ unit derived from malonyl-CoA the chain grows with two carbons until an average length of 16 carbons is reached. However, each reaction cycle includes two reduction steps, each requiring the redox co-factor NADPH [19]. Therefore, another metabolic pathway leading towards the synthesis of acetyl-CoA was investigated.

The phosphoketolase (PHK) pathway was described previously as potential alternative carbon route for different industrially relevant metabolites due to the increase of acetyl-CoA production with NADPH as co-product [20-22]. The carbon flow leading towards the PHK pathway starts with the conversion of glucose to glucose-6-phosphate in the Embden-Meyerhof-Parnas (EMP) pathway followed by conversion of glucose-6phosphate in three reactions into ribulose-5-phosphate in the oxidative part of the pentose phosphate pathway (PPP). Byproducts during these reactions are two moles of NADPH and one mole of $\mathrm{CO}_{2}$ per mole of glucose. The next metabolite in the pathway, xylulose-5-phosphate, represents the precursor of the PHK pathway as it can be converted to acetyl-phosphate and glyceraldehyde3 -phosphate. This reaction is catalyzed by xylulose-5phosphate phosphoketolase encoded by xpkA in Aspergillus nidulans. During the second step of the PHK pathway, acetyl phosphate can either directly be converted into acetyl-CoA or indirectly with acetate as intermediate. Direct conversion of acetyl phosphate into acetyl-CoA in Bacillus subtilis is catalyzed by phosphotransacetylase encoded by the gene pta [23]. The formation of acetate and ATP from acetyl phosphate is performed by acetate kinase, encoded by the gene ack in A. nidulans [24,25]. The metabolic pathway is shown in Figure 1A.

The PHK pathway was first reported in heterofermentative and facultative homofermentative lactic acid bacteria, in bifidobacteria and sporadic in other microorganism, like xylose fermenting yeasts [26]. It has been demonstrated that $S$. cerevisiae has the ability to functionally express phosphotransacetylase from B. subtilis as well as phosphoketolase and acetate kinase from $A$. nidulans [20,27]. The fact that more NADPH is formed when glucose is metabolized via the PPP instead of the EMP pathway makes the combination of PPP and PHK pathway an interesting alternative for FAEE production. The demand of NADPH for lipid synthesis might create the driving force for carbon to flow through the PHK pathway. The reaction equations in Figure $1 \mathrm{~B}$ show that glucose catabolism through the PHK pathway would reduce the net NADPH demand for FAEE production. 


\section{A}

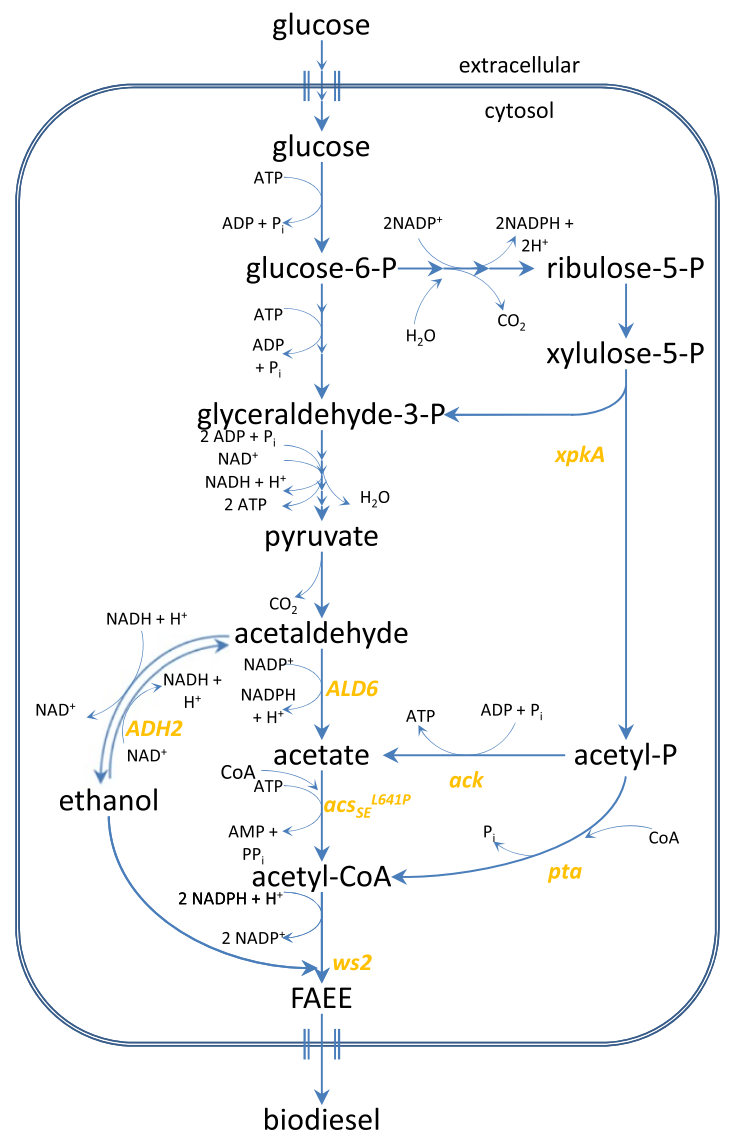

B

FAEE production via EMP pathway
9 glucose $+18 A D P+18 P_{i}+16 N A D^{+}+16 N A D P H \rightarrow 2 F A E E(2 e t h a n o l+2 a c y l C o A(16 C))+$
$18 C O_{2}+2 A T P+16 A M P+16 P P_{i}+16 N A D H+18 H_{2} O+16 N A D P^{+}$

FAEE production via PPP and PHK pathway genes xpkA and ack

9 glucose $+18 A D P+18 P_{i}+7 N A D^{+}+7 N A D P H$

$\rightarrow 2 F A E E(2$ ethanol $+2 F A(16 C))+18 C_{2}+2 A T P+16 A M P+16 P P_{i}$

$+7 N A D P^{+}+7 N A D H$

FAEE production via PPP and PHK pathway genes $x p k A$ and pta

$$
\begin{aligned}
9 \text { glucose }+9 A D P & +9 P_{i}+7 N A D^{+}+7 N A D P H \\
& \rightarrow 2 F A E E(2 \text { ethanol }+2 F A(16 C))+18 C O_{2}+2 A T P+7 A M P+7 P P_{i} \\
& +7 N A D P^{+}+7 N A D H
\end{aligned}
$$

Figure 1 Overview of metabolic engineering strategies. A. Schematic metabolic pathways describing the (over-)expression of the ethanol degradation pathway and the phosphoketolase pathway. The genes of interest are: xpkA (xylulose-5-phosphate phosphoketolase), ack (acetate kinase), pta (phosphotransacetylase), ADH2 (alcohol dehydrogenase 2), ALD6 (acetaldehyde dehydrogenase), acS SE ${ }^{\text {L64IP }}$ (acetyl-COA synthetase) and ws2 (wax ester synthase). B. Stoichiometric equations for the conversion of glucose to the product of interest, FAEE, via either the EMP pathway or the combination of PP and PHK pathway. For convenience it was assumed that the acyl-CoA chain was unsaturated and 16 carbons long. Abbreviations: ADP (adenosine diphosphate), NAD (nicotinamide adenine dinucleotide), NADPH (nicotinamide adenine dinucleotide phosphate hydrogenase), ATP (adenosine triphosphate), NADH (nicotinamide adenine dinucleotide hydrogenase), NADP ${ }^{+}$(nicotinamide adenine dinucleotide phosphate). 
Here, the research goal was the improvement of a $S$. cerevisiae FAEE cell factory. In more detail, ws 2 from $M$. hydrocarbonoclasticus [4] was expressed in combination with the two different pathways mentioned above, i.e. the 'ethanol degradation' pathway responsible for supplying precursor acetyl-CoA and the heterologous phosphoketolase pathway supplying both acetyl-CoA and redox co-factor NADPH. The results of expressing the two pathways were compared in terms of physiological properties and FAEE production of the recombinant strains.

\section{Materials and methods}

\section{Strains}

All plasmid constructions were performed with $E$. coli strain DH5 $\alpha$ [28]. Yeast strain S. cerevisiae CEN.PK 11311C (MATa MAL2-8 ${ }^{c}$ SUC2 ura3-52 his3- $\Delta 1$ ) was kindly provided by P. Kötter, University of Frankfurt, Germany. $S$. cerevisiae CB2I20 is a derivative of CEN.PK 113-5D (MATa MAL2-8 ${ }^{C}$ SUC2 ura3-52) and contains multiple ws 2 chromosomal integrations. This strain has been used for stable expression of $w s 2$ and expression of genes encoding endogenous acyl-CoA binding protein $(A C B 1)$ and a bacterial $\mathrm{NADP}^{+}$-dependent glyceraldehyde-3-phosphate dehydrogenase $(\operatorname{gap} N)$ (unpublished).

\section{Media and growth conditions}

E. coli cells were cultured at $37^{\circ} \mathrm{C}$ and $200 \mathrm{rpm}$ in lysogeny broth (LB) [29] containing $80 \mathrm{mg} \mathrm{l}^{-1}$ ampicillin when needed. $S$. cerevisiae strains were cultured at $30^{\circ} \mathrm{C}$ and $150 \mathrm{rpm}$ in synthetic dextrose (SD) medium containing $20 \mathrm{~g} \mathrm{l}^{-1}$ glucose, $6.7 \mathrm{~g} \mathrm{l}^{-1}$ yeast nitrogen base without amino acids (YNB-AA) (Formedium, Hunstanton, UK), and complete supplement mixture $\left(0.750 \mathrm{~g} \mathrm{l}^{-1}\right.$; CSM, Formedium) lacking uracil and histidine.

\section{Plasmid construction}

Plasmids containing a HIS3 selection marker were derived from episomal 2-micron plasmids pIYC04 and pIYC08. The construction of the plasmids was described by Chen et al. [12,13]. The codon optimized ws2 gene from $M$. hydrocarbonoclasticus (Menlo Park, CA, USA) [4] was amplified with forward primer CTTCAAACTAGTAAAA CAATGAAGAGATTAGGTACTCTAGACG and reverse primer CTTCTTGAGCTCTTACTTTCTAGTACGGGCA CG attaching restriction sites SpeI and SacI (marked italic) to the gene. This fragment was cloned into a multi-cloning site on pIYC04 resulting in a plasmid containing the ws 2 gene under control of the TEF1 promoter and the $A D H 1$ terminator. This plasmid was verified by sequencing (Eurofins MWG Operon, Ebersberg, Germany) and named pBDJ01. pBDJ02 was constructed from plasmids pIYC08 and pBDJ01, which were both restricted with $S a c I$ and SpeI. The insert from pBDJ01 containing the ws2 gene was cloned into the pIYC08 backbone containing ADH2, ALD6 and $\operatorname{acS}_{S E}{ }^{L 641 P}$. pBDJ02 finally contained four genes: ws2, ALD6, ADH2 and $a c s_{S E}{ }^{L 641 P}$. The construction of an additional plasmid (pIYC09) containing genes ALD6, $A D H 2$ and $\operatorname{acs}_{S E}{ }^{\text {L641P }}$ was described by Chen et al. [30]. Plasmid pSP-GM2 [31,32] was used as a backbone for the construction of plasmids that contained the URA3 selection marker. The ws 2 gene was restricted with NotI/SacI from pBdJ02 and then ligated into the NotI/ $\mathrm{SacI}$ sites of vector pSP-GM2 to construct pSP-B2N. The construction of plasmid $\mathrm{pMPa}$ containing genes $x p k A$ and ack was described previously [20]. Plasmid pMPp was constructed accordingly with the difference that it contained pta instead of ack. All plasmids were confirmed by sequencing (Eurofins). A list of all plasmids is shown in Table 1.

\section{Strain construction and transformation}

The different yeast strains constructed are listed in Table 2. Transformations were performed by following the lithium acetate/single-stranded carrier DNA/polyethylene glycol method [33].

\section{Culturing}

The different S. cerevisiae strains were stored in $15 \%$ glycerol at $-80^{\circ} \mathrm{C}$ before they were pre-cultured in $5 \mathrm{ml}$ selective $\mathrm{SD}$ medium at $30^{\circ} \mathrm{C}$ and $200 \mathrm{rpm}$. Thereafter, the strains were grown in $500 \mathrm{ml}$ shake flasks containing $50 \mathrm{ml}$ selective SD-medium starting with an optical density $\left(\mathrm{OD}_{600}\right)$ of 0.01 . Cultures were grown for up to 100 hours at $30^{\circ} \mathrm{C}$ and $150 \mathrm{rpm}$.

\section{Analysis of physiological parameters}

While culturing, samples were taken regularly (every 3-4 h). The cell growth was measured with a Genesys 20 spectrophotometer (Thermo Fisher Scientific Inc., Waltham, MA, USA) by determining the optical density at $600 \mathrm{~nm}$. The concentration of remaining substrate and formation of byproducts (ethanol and glycerol) was analyzed using filtered samples $(0.2 \mu$ m nylon membrane) by high performance liquid chromatography (HPLC; Dionex Ultimate $3000 \mathrm{HPLC}$ system Dionex Softron GmbH, Germering, Germany) equipped with an Aminex HPX-87H column (Bio-Rad, Hercules, CA, USA) at $65^{\circ} \mathrm{C}$ and fed with a mobile phase of $5 \mathrm{mM} \mathrm{H}_{2} \mathrm{SO}_{4}$ at a flow rate of $0.6 \mathrm{ml} \mathrm{min}^{-1}$. Glucose, glycerol and ethanol were measured with a Shodex RI-101 refractive index detector (Showa Denko, Tokyo, Japan).

\section{Sample preparation for quantification of FAEEs}

After 100 hours of culturing the remaining biomass was centrifuged and washed with MQ water thrice before being freeze-dried (Christ Alpha 2-4 LSC, Shropshire, UK). Further sample treatment was performed with a known amount of cell dry weight (CDW) and $20 \mu \mathrm{g}$ of 
Table 1 List of plasmids used in this study

\begin{tabular}{|c|c|c|c|}
\hline $\begin{array}{l}\text { Plasmid } \\
\text { name }\end{array}$ & Genes & $\begin{array}{c}\text { Marker } \\
\text { gene }\end{array}$ & Source \\
\hline pIYC04 & - & HIS3 & {$[12]$} \\
\hline pBdJ01 & ws2 & HIS3 & This study \\
\hline pICY09 & $A L D 6, A D H 2, a_{S E}{ }_{S 641 P}$ & HIS3 & [13] \\
\hline pBdJ02 & ws2, ALD6, ADH2, aCSSE ${ }^{L 641 P}$ & HIS3 & This study \\
\hline pSP-GM2 & - & URA3 & [31] \\
\hline pSPB2N & ws2 & URA3 & Shi et al., submitted \\
\hline $\mathrm{pMPa}$ & $x p K A, a c k$ & URA3 & [21] \\
\hline pMPp & xpKA, pta & URA3 & This study \\
\hline
\end{tabular}

heptadecanoic acid ethyl ester (17:0) as internal standard (IS). This sample was mixed with $7 \mathrm{ml} \mathrm{CHCl}{ }_{3}: \mathrm{MeOH}$ $(2: 1, \mathrm{v} / \mathrm{v})$ in an extraction tube (Pyrex borosilicate glass $16 \times 100 \mathrm{~mm}$ ) and flushed with nitrogen gas until all air was removed before total lipids were extracted from the sample by a microwave-assisted method described previously [34]. Next, $1.7 \mathrm{ml} \mathrm{NaCl}(0.73 \%$, w/v) was added to the sample and vortexed vigorously. Centrifugation at $3000 \mathrm{rpm}$ for $5 \mathrm{~min}$ resulted in a phase separation. The organic (lower) phase was transferred into a clean tube for vacuum evaporation using a miVac concentrator (Genevac, Ipswich, UK). The sample was re-suspended in $50 \mu \mathrm{l} \mathrm{CHCl}{ }_{3}: \mathrm{MeOH}(2: 1, \mathrm{v} / \mathrm{v})$, loaded onto a thin layer chromatographic (TLC) Silica gel 60 F254plate (Merck, Darmstadt, Germany) and separated using a mobile phase of heptane, 2-propanol and acetic acid with a ratio of 95:5:1 (v/v/v) [4]. Free fatty acids, triacylglycerols (TAGs), sterol esters and FAEEs were identified using a standard solution containing heptadecanoic acid, glyceryl triheptadecanoate, cholesteryl palmitate and heptadecanoic acid ethyl ester (Sigma-Aldrich, St. Louis, MO, USA). Visualization was performed under ultraviolet radiation after spraying with $0.05 \%$ 2,7-dichlorofluorescein in ethanol. The spot of the TLC plate corresponding to FAEEs was scraped off with a razor blade and collected in a tube containing a mix of $3 \mathrm{ml}$ hexane, $2 \mathrm{ml}$ methanol and $2 \mathrm{ml} \mathrm{MQ}$ water. The tube was vigorously vortexed, and after centrifugation at $3000 \mathrm{rpm}$ for $5 \mathrm{~min}$, the upper layer was transferred to a clean tube. This solution, containing the FAEEs, was dried by vacuum evaporation using a miVac concentrator (Genevac, Ipswich, UK) before the sample was finally dissolved in $1 \mathrm{ml}$ hexane.

\section{GC-MS analysis}

The equipment for separating and quantifying FAEEs included a Focus GC ICQ single quadruple GC-MS from Thermo Fisher Scientific with a Zebron (ZB-WAX) GC column with $30 \mathrm{~m} \times 0.25 \mathrm{~mm}$ internal diameter and $0.25 \mu \mathrm{m}$ film thickness (Phenomenex, Macclesfield, UK). The precise GC-MS conditions, compound identification and quantification conditions have previously been described [4].

\section{Results}

FAEE production in strains expressing the ethanol degradation pathway

The production of FAEEs requires a large amount of acetyl-CoA and therefore it was a straight forward idea to metabolically engineer the native pathway in S. cerevisiae to increase the flux towards cytosolic acetyl-CoA synthesis. Ethanol, the second metabolite for synthesis of FAEEs, forms the major byproduct during FAEE production in S. cerevisiae. To re-channel the carbon flux towards the synthesis of acetyl-CoA, Adh2 and Ald6, which respectively catalyze the conversion of ethanol to acetaldehyde and acetaldehyde to acetate, were overexpressed together with the heterologous $\operatorname{acs}_{S E}{ }^{L 641 P}$, which encodes a de-regulated acetyl-CoA synthetase that catalyzes the conversion of acetate to acetyl-CoA. Details about the metabolic

Table 2 Strains used in this study

\begin{tabular}{|c|c|c|c|}
\hline Name & Genetic background & HIS3 based plasmid & URA3 based plasmid \\
\hline \multicolumn{4}{|c|}{ Reference strain } \\
\hline BdJref & CEN.PK 113-11C & plYC04 (---) & pSP-GM2 (---) \\
\hline \multicolumn{4}{|c|}{ Phosphoketolase strains } \\
\hline BdJ01 & CEN.PK 113-11C & pBdJ01 (ws2) & pSP-GM2 (---) \\
\hline $\mathrm{BdJ02}$ & CEN.PK 113-11C & pBdJ01 (ws2) & $\mathrm{pMPa}(x p k A, a c K)$ \\
\hline BdJ03 & CEN.PK 113-11C & pBdJ01 (ws2) & $\operatorname{pMPp}(x p k A, p t a)$ \\
\hline BdJ04 & CB2I20 & - & pSP-GM2 (---) \\
\hline BdJ05 & CB2120 & - & $\mathrm{pMPa}(x p k A, a c k)$ \\
\hline BdJ06 & CB2120 & - & pMPp (xpkA, pta) \\
\hline \multicolumn{4}{|c|}{ Ethanol degradation strains } \\
\hline BdJ07 & CEN.PK 113-11C & plYC04 (---) & pSPB2N (ws2) \\
\hline BdJ08 & CEN.PK 113-11C & $\mathrm{pBdJ02}$ (ws2, ALD6, ADH2, aCsSE & pSP-GM2 (---) \\
\hline BdJ09 & CEN.PK $113-11 C$ & $\operatorname{plYC09}\left(A L D 6, A D H 2, \operatorname{acSSE}_{S E}^{L 641 P}\right)$ & pSPB2N (ws2) \\
\hline
\end{tabular}


pathway and the strain construction are shown in Figure 1 and Table 2, respectively.

In Figure 2, it can be observed that the introduction of a wax ester synthase resulted in a FAEE yield of $133 \pm$ $113 \mu \mathrm{g} \mathrm{gCDW}^{-1}$ (strain BdJ07). Up-regulation of ALD6, $A D H 2$ and $a_{C S_{S E}}{ }^{L 641 P}$, expressed from the same plasmid, led to a yield of $408 \pm 270 \mu \mathrm{gCDW}^{-1}$ (strain BdJ08), a 3-fold improvement compared to strain BdJ07, which however lacks statistical significance (p-value: 0.08). The strain showed a large variation of the FAEE yield in the different clones measured, which might be caused by variations in copy number of the relatively large plasmid. Therefore, a similar strain was constructed, reducing the plasmid size by expressing the genes on two different plasmids. This strain, BdJ09, in which ws 2 and the three genes $A L D 6, A D H 2$ and $a c S_{S E}{ }^{L 641 P}$ were expressed from separate plasmids resulted in a FAEE yield of $359 \pm$ $128 \mu \mathrm{g} \mathrm{gCDW}^{-1}$. This modified strain showed a 2.7 fold improvement compared to strain BdJ07, which only expressed ws2 (p-value: 0.03), and also showed a lower clonal variation than strain BdJ08.

\section{FAEE production in strains expressing the PHK pathway} Introduction of the PHK pathway has the potential to increase the yield of FAEEs in $S$. cerevisiae due to its ability to generate two moles of NADPH per mole of glucose, which would provide additional NADPH for acyl-CoA synthesis. Therefore, two genes, $x p k A$ and ack, both descending from $A$. nidulans, were expressed to catalyze, respectively, the conversion of xylulose-5phosphate to acetyl phosphate and glyceraldehyde-3phosphate and acetyl phosphate to acetate with the gain of one ATP. A second strain was constructed, in which the gene pta from B. subtilis replaced ack. Pta converts acetyl phosphate directly to acetyl-CoA. Both strains were compared with strain BdJ01 containing solely the ws 2 gene and to a reference strain (BdJref) containing the empty reference plasmids. Figure 3 shows

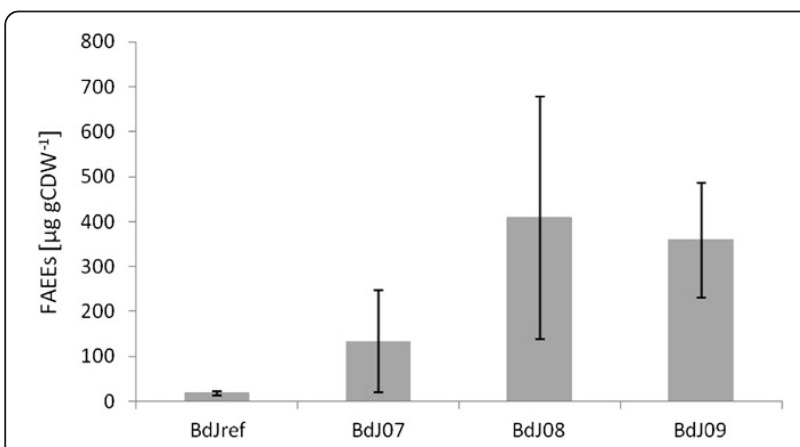

Figure 2 FAEE yield $\left(\mu \mathrm{g} \mathrm{gCDW}{ }^{-1}\right.$ ) of investigated S. cerevisiae strains expressing the ethanol degradation pathway. Biological triplicates of the strains were investigated. The experiment was performed twice and standard deviations are indicated. the quantification of total FAEEs of the described $S$. cerevisiae strains. The expression of $x p k A$, ack and $w s 2$ (strain BdJ02) resulted in a FAEE yield of $28 \pm 3.5 \mu \mathrm{g}$ $\mathrm{gCDW}^{-1}$ which is 1.5 times higher than for the strain only expressing ws2 (BdJ01). However, the difference between the two strains showed insufficient statistical significance (p-value: 0.10). The expression of $x p k A$, pta and ws2 (BdJ03) led to a FAEE yield of $105 \pm 30 \mu \mathrm{g}$ $\mathrm{gCDW}^{-1}$ which was 5.7 fold higher than the strain with solely ws 2 expression (p-value: 0.03 ) and a 3.7 times higher FAEE yield than the strain expressing $x p k A$, ack and ws2 (p-value: 0.05 ).

Due to the relatively low yield of the strains described above and because of presumed fluctuating plasmid stability during repetitive experiments, the PHK pathway was also expressed in strain CB2I20, a strain with multiple chromosomal ws2 integrations (Shi et al., submitted). These strains are listed in Table 2. It was also hypothesized that multiple expression of the ws 2 gene might contribute to a stronger carbon-pull through the PHK pathway. Expression of $x p k A$ and $p t a$ in strain CB2I20 (BdJ06) resulted in $4670 \pm 379 \mu \mathrm{g} \mathrm{gCDW}^{-1}$ (p-value: 0.02 ), a 1.6 fold higher yield of FAEEs compared to the reference strain CB2I20 (BdJ04), whereas the expression of $x p k A$ and ack in CB2I20 (BdJ05) improved the final yield of FAEEs to $5100 \pm 509 \mu \mathrm{g} \mathrm{gCDW}^{-1}$ (p-value: 0.01 ), which is a 1.7 times improvement compared to BdJ04 (Figure 4). It could also be observed that the production levels of FAEEs in strains BdJ04-BdJ06 were stable and reproducible.

\section{Physiological data}

Wild type $S$. cerevisiae is a fast growing and robust microorganism. However, the genetic modifications leading to improved FAEE production could have an impact on its physiology. Cell growth, glucose consumption as well as ethanol formation and consumption were therefore monitored during shake flask cultivations (Table 3 and

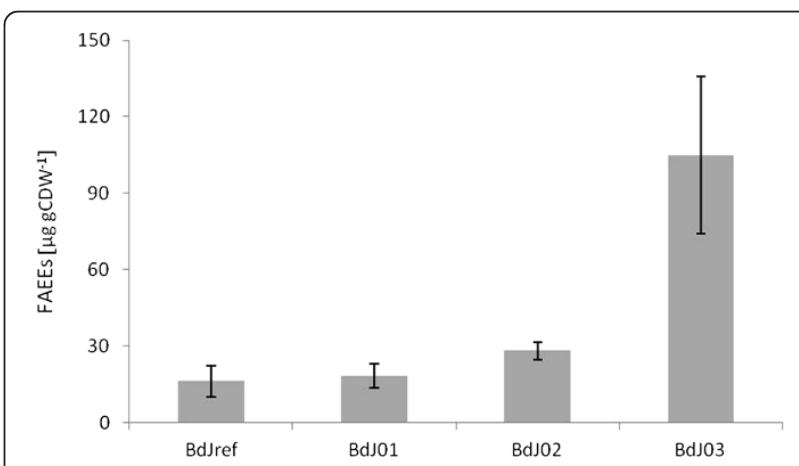

Figure 3 FAEE yield $\left(\mu \mathrm{g} \mathrm{gCDW}{ }^{-1}\right.$ ) of investigated S. cerevisiae strains expressing a heterologous PHK pathway. Biological triplicates of the strains were investigated. The standard deviations are indicated. 
Figure 5). The final biomass of the engineered strains was reduced compared to the reference strain (BdJref) and also the maximum specific growth rate $\left(\mu_{\max }\right)$ for both PHK pathway expressing strains and ethanol degradation pathway engineered strains showed a reduction of up to $40 \%$ (BdJ08). The maximal glucose consumption rate ( $\mathrm{q}_{\text {smax }}$ ) was only slightly reduced for the engineered strains if compared to the reference strain. The most noticeable physiological change between the strains expressing the PHK pathway and the strains engineered for increased ethanol degradation was a slower maximal consumption rate of ethanol ( $\mathrm{q}_{\text {ethmax }}$ ) for the latter (strains BdJ07-BdJ09). Especially the strains overexpressing genes $A D H 2, A L D 6, \operatorname{acs}_{S E}{ }^{L 641 P}$ and $w s 2$ showed a reduced ethanol consumption rate of, respectively, 45\% (BdJ08) and $70 \%$ (BdJ09) compared to the reference strain.

\section{Discussion}

The production of FAEEs in yeast $S$. cerevisiae could form an important contribution to the development of sustainable diesel transportation fuels in the future. However, to turn $S$. cerevisiae into an efficient FAEE producer instead of an ethanol producer, its metabolism requires further engineering. FAEEs are synthesized from acyl-CoA and ethanol and the formation of acyl-CoA in turn is dependent on precursors acetyl-CoA and there from derived malonyl-CoA. Acyl-CoA formation also requires a large amount of co-factor NADPH. In this study, two different metabolic engineering strategies were applied to increase the supply of carbon and co-factor NADPH for production of the FAEE precursor acyl-CoA.

The two main metabolic reactions in S. cerevisiae to produce NADPH during growth on glucose are the conversion of acetaldehyde to acetate by acetaldehyde dehydrogenase and the first steps in the pentose phosphate pathway (catalyzed by glucose-6-phosphate dehydrogenase and 6-phosphogluconate dehydrogenase). Grabowska et al. demonstrated this by constructing a $S$. cerevisiae mutant with deletions in ZWF1 (encoding glucose-6-phosphate dehydrogenase) and ALD6 (encoding cytosolic aldehyde dehydrogenase), which was not viable on glucose [35]. Later, an additional source of NADPH was identified during growth on lactate, namely cytosolic isocitrate dehydrogenase (Idp2) [36].

In this study, it was found that expression of the phosphoketolase pathway in S. cerevisiae could re-channel carbon flux through the oxidative part of the PPP towards the precursors acetyl-CoA and malonyl-CoA for synthesis of acyl-CoA. As a consequence, two molecules of the NADPH are being produced for each sugar molecule passing through the PPP. As was demonstrated by Papini et al. the expression of genes $x p k A$ and ack showed a functional carbon flux through the PHK pathway and as shown in this study the expression of genes $x p k A$, ack and ws 2 resulted in a yield of FAEEs $50 \%$ higher than in a strain not expressing the PHK pathway [20]. It was also shown that the expression of $x p k A$, ack resulted in an improved polyhydroxybutyrate (PHB) producing strain [22]. Papini et al. could not demonstrate the functionality of the PHK pathway using phosphotransacetylase gene pta (instead of ack), which would result in a direct conversion of acetyl phosphate to acetyl-CoA. However, in their study they did not insert a pull of acetyl-CoA for a specific product, whereas our result clearly shows that expression of this enzyme combination results in improved FAEE production (minimal 60\% improvement), which

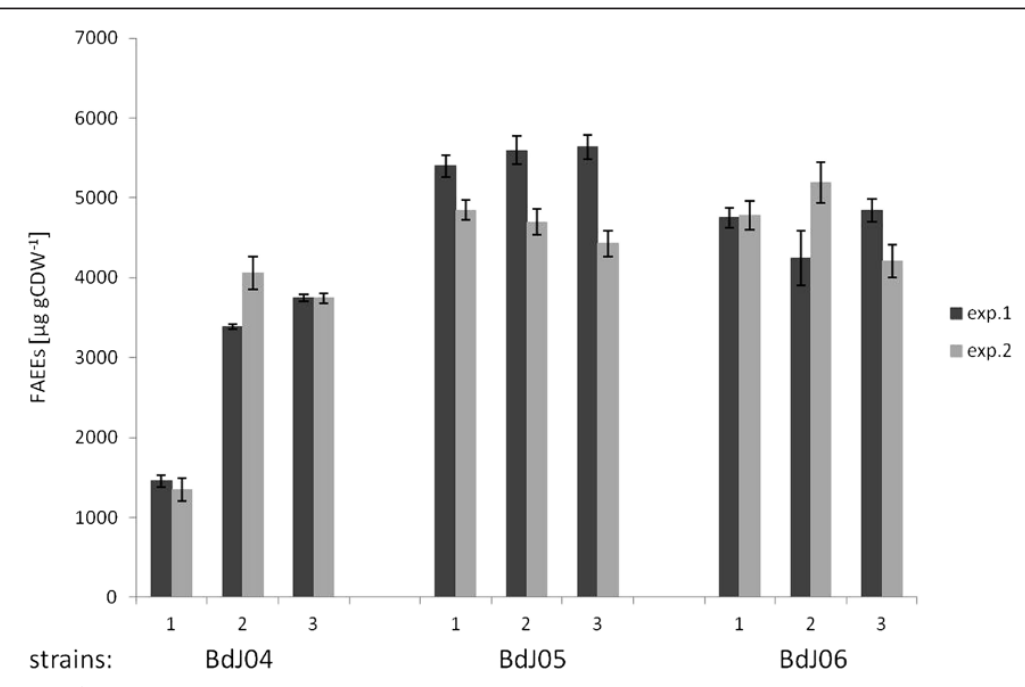

Figure 4 FAEE yield $\left(\mu \mathrm{g} \mathrm{gCDW}{ }^{-1}\right)$ of investigated $S$. cerevisiae strains containing multiple ws 2 chromosomal integrations and expressing a heterologous PHK pathway. Triplicate clones of each strain were investigated (described by 1,2 and 3). The experiment was performed twice (light and dark columns) and standard deviations are indicated. 
Table 3 Physiological characteristics of modified strains

\begin{tabular}{|c|c|c|c|c|c|c|}
\hline Strain & $\begin{array}{c}\text { (Over)expressed genes } \\
\text { (HIS3 plasmid) \& (URA3 plasmid) }\end{array}$ & $\begin{array}{l}\text { Final FAEE yield } \\
\left(\mu \mathrm{g} \mathrm{gCDW} W^{-1}\right)\end{array}$ & $\begin{array}{l}\text { Maximal specific } \\
\text { growth rate }\left(\mu_{\max }\right)\end{array}$ & $\begin{array}{l}\text { Final biomass } \\
(\text { OD 600) }\end{array}$ & $\begin{array}{l}\text { Glucose consumption } \\
\text { rate }\left(\mathbf{q}_{\text {smax }}\right)\end{array}$ & $\begin{array}{l}\text { Ethanol consumption } \\
\text { rate }\left(q_{\text {ethmax }}\right)\end{array}$ \\
\hline BdJref & $-\&--$ & $16.3 \pm 3.5$ & $0.190 \pm 0.010$ & $5.84 \pm 0.14$ & $-1.534 \pm 0.054$ & $-0.204 \pm 0.015$ \\
\hline \multicolumn{7}{|c|}{ Phosphoketolase pathway } \\
\hline BdJ01 & ws2 \& -- & $18 \pm 4.8$ & $0.159 \pm 0.006$ & $5.51 \pm 0.16$ & $-1.448 \pm 0.057$ & $-0.200 \pm 0.021$ \\
\hline $\mathrm{BdJ02}$ & ws2 \& xpkA, ack & $28.0 \pm 3.5$ & $0.168 \pm 0.009$ & $5.27 \pm 0.13$ & $-1.357 \pm 0.084$ & $-0.208 \pm 0.016$ \\
\hline BdJ03 & ws2 \& xpkA, pta & $105 \pm 30$ & $0.136 \pm 0.025$ & $4.55 \pm 0.11$ & $-1.357 \pm 0.078$ & $-0.197 \pm 0.014$ \\
\hline \multicolumn{7}{|c|}{ Ethanol degradation pathway } \\
\hline BdJ07 & - \&ws2 & $133 \pm 113$ & $0.192 \pm 0.003$ & $5.14 \pm 0.22$ & $-1.403 \pm 0.035$ & $-0.174 \pm 0.003$ \\
\hline BdJ08 & ws2, ALD6, ADH2, aCSSE ${ }_{S E}^{\text {64PP }} \&-$ & $408 \pm 270$ & $0.115 \pm 0.026$ & $3.97 \pm 0.42$ & $-1.265 \pm 0.365$ & $-0.113 \pm 0.002$ \\
\hline BdJ09 & $A L D 6, A D H 2, a_{S E}^{L 647 P} \& w S 2$ & $359 \pm 128$ & $0.136 \pm 0.035$ & $3.67 \pm 1.82$ & $-1.208 \pm 0.495$ & $-0.061 \pm 0.023$ \\
\hline
\end{tabular}

strongly indicates that the enzymes are active. We therefore conclude that the expression of ack from $A$. nidulans or pta from B. subtillis combined with expression of $x p k A$ from $A$. nidulans and ws 2 from $M$. hydrocarbonoclasticus represents a successful strategy for increasing FAEE production by $S$. cerevisiae.

The second strategy, the ethanol degradation pathway, was based on the native acetyl-CoA supply in the cytosol of $S$. cerevisiae. Chen et al. investigated the physiological effect of some major enzymes relevant for acetyl-CoA metabolism on different carbon sources in S. cerevisiae [12]. Besides being regulated at the transcriptional and post-translational level, yeast acetyl-CoA synthetases Acs1 and Acs2 also show differential subcellular localization $[12,37]$. It was shown that the posttranslationally regulated acetyl-CoA synthetase in S. enterica is prevented from acetylation by a point mutation substituting leucine for proline at position 641 of the enzyme and maintaining it in its active state [18]. This enzyme variant encoded by $\operatorname{acs}_{S E}{ }^{L 641 P}$ was previously overexpressed together with $A L D 6$, mainly responsible for the metabolic reaction to form acetate from acetaldehyde, in S. cerevisiae for highlevel production of amorphadiene [17]. This successful strategy had been improved by additional overexpression of $A D H 2$ and ERG10 for the production of $\alpha$-santalene which resulted in a 1.75 times higher production than the reference strain (without overexpression of the four genes) and a $25 \%$ increase in titer compared to the strategy introduced by Shiba et al. [17]. The pull-push strategy, pushing the carbon flow down to acetyl-CoA by overexpression of $A D H 2$, ALD6 and $\operatorname{acs}_{S E}^{L 641 P}$ and pulling it towards the product by over expression of ERG10 was also applied in the production of polyhydroxybutyrate (PHB) in $S$. cerevisiae [38]. A 16-fold improvement of $\mathrm{PHB}$ production was detected compared to the reference strain. In this study, the wax ester synthase was used to pull the carbon flow towards production of FAEEs. Comparable to the previously performed studies described, we here found the improvement of product formation to be 3 -fold compared with the reference strain.
It remains important to consider the different platforms for gene expression in $S$. cerevisiae. In general, the expression can occur from plasmids or genes can be integrated into the chromosomes. Here both engineered pathways were expressed on 2-micron plasmids resulting in a high copy numbers and therefore high expression. However, expression of several genes on 2-micron plasmids results in large plasmids (pBdJ02 > $15 \mathrm{~kb}$ ). Plasmid size as well as promoter strengths influences the stability of the plasmid and therefore the gene expression when propagated in $S$. cerevisiae [39-41]. We indeed saw a high clonal variation in some of our plasmid based strains. On the other hand, integration of genes into the chromosomes results in stable expression levels as was clearly demonstrated by Jensen et al. [42]. Strain CB2I20, which was used for expression of the PHK pathway, contained multiple (1-6) integrated copies of the ws 2 gene which resulted in stable production levels of FAEE with a 10 fold higher yield (Shi S, Valle-Rodríguez J, Siewers V, Nielsen J: Engineering of chromosomal wax ester synthase integrated Saccharomyces cerevisiae mutants for improved biosynthesis of fatty acid ethyl esters, submitted). The high yield in combination with stable expression levels reached by multiple integrated copies is especially valuable for industrial yeast strains.

Further, it seems unlikely that FAEE is toxic to $S$. cerevisiae at the concentrations produced here. There was no negative effect on cell growth when $1 \mathrm{~g} / \mathrm{l}$ of myristic acid ethyl esters were added to the medium (data not shown). Therefore, the reduced ethanol consumption rates observed (45\% in BdJ08 and 70\% in BdJ09 during the ethanol degradation pathway might most likely be explained by the rapid conversion of the ethanol to cytosolic acetylCoA which hence reduces the amount of ethanol that can be oxidized in the mitochondria. In general, it might be a presumable assumption that the metabolic changes were caused by the overexpression of several genes.

Thus, in conclusion we found that both strategies for improving the supply of cytosolic acetyl-CoA and NADPH, i.e. overexpression of the heterologous PHK pathway or the overexpression of the ethanol degradation pathway, 

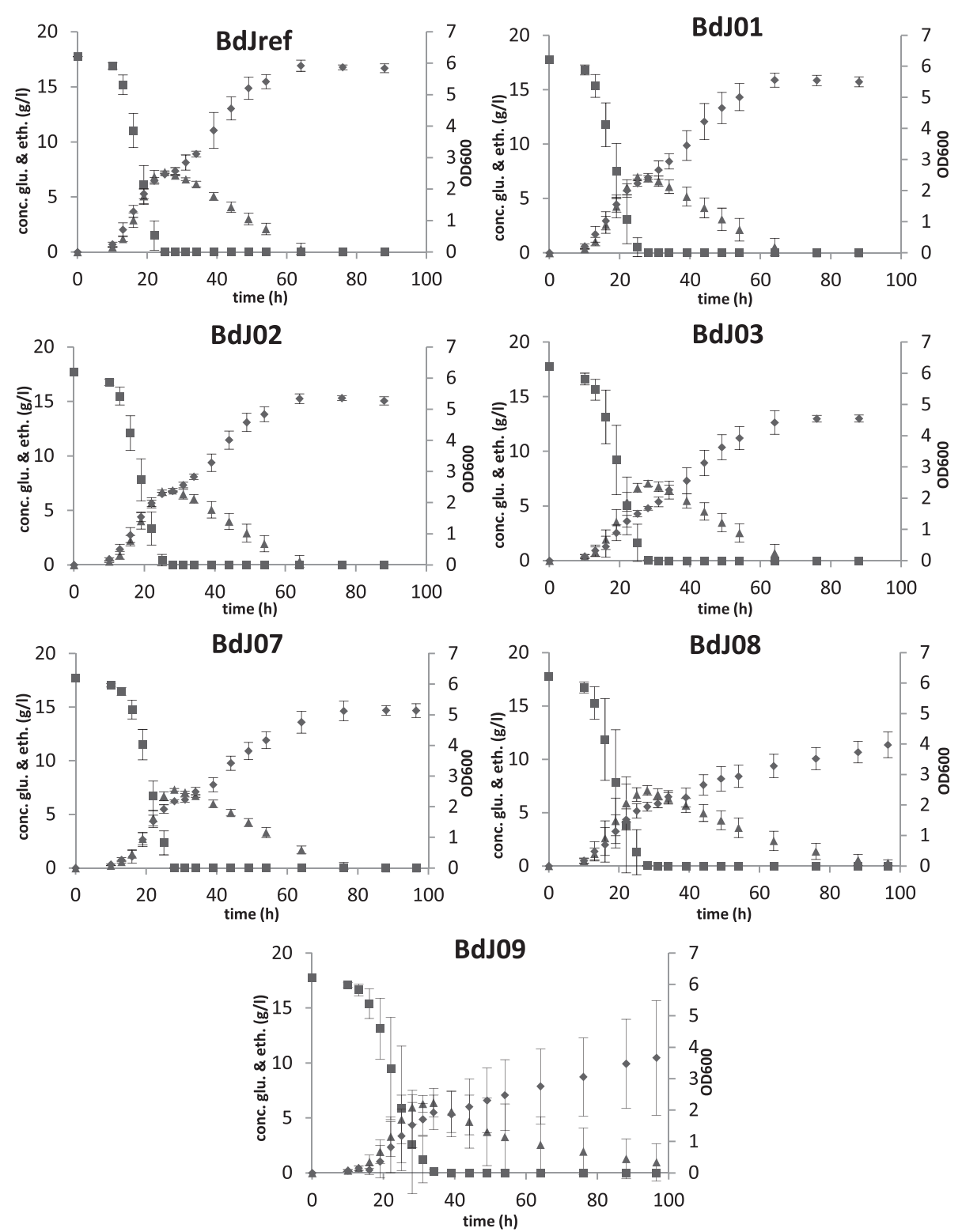

Figure 5 Cell growth and substrate consumption of recombinant strains during shake flask cultivation. The strains BdJref and BdJ01-BdJ06 were analyzed in biological triplicates. The glucose concentration $\left(\mathrm{gl}^{-1}\right)$ is indicated by squares, the ethanol concentration $\left(\mathrm{g} \mathrm{I}^{-1}\right)$ is indicated by triangles and cell growth (OD 600) is shown by diamonds.

resulted in improvement in the production of FAEEs, and hence form the basis for engineering an efficient cell factory for production of this biodiesel component.

\section{Acknowledgement}

The research leading to these results has been made possible by the Knut and Alice Wallenberg Foundation, Vetenskapsrådet, FORMAS, and

Ångpanneföreningens Forskningsstiftelse.

\section{Competing interest}

The author's declare that they have no competing interests.

\section{Authors' contributions}

BWDJ performed all the experiments and took part in designing the study, evaluating the results and writing the manuscript. SS, VS and JN took part in designing the study, evaluating the results and writing the manuscript. This manuscript has been approved by all the authors listed.
Author details

'Department of Chemical and Biological Engineering, Chalmers University of Technology, Kemivägen 10, Göteborg SE-412 96, Sweden. ${ }^{2}$ Institute of

Chemical and Engineering Sciences, A-Star, 31, Biopolis Way, \#01-01 Nanos, Singapore 138669, Singapore.

Received: 8 January 2014 Accepted: 5 March 2014 Published: 12 March 2014 


\section{References}

1. de Jong B, Siewers V, Nielsen J: Systems biology of yeast: enabling technology for development of cell factories for production of advanced biofuels. Curr Opin Biotechnol 2012, 23:624-630.

2. Kalscheuer R, Luftmann $H$, Steinbuchel A: Synthesis of novel lipids in Saccharomyces cerevisiae by heterologous expression of an unspecific bacterial acyltransferase. Appl Environ Microbiol 2004, 70:7119-7125

3. Kalscheuer R, Stolting T, Steinbuchel A: Microdiesel: Escherichia coli engineered for fuel production. Microbiology 2006, 152:2529-2536.

4. Shi S, Valle-Rodriquez JO, Khoomrung S, Siewers V, Nielsen J: Functional expression and characterization of five wax ester synthases in Saccharomyces cerevisiae and their utility for biodiesel production. Biotechnol Biofuels 2012, 5:7.

5. Barney BM, Wahlen BD, Garner E, Wei JS, Seefeldt LC: Differences in substrate specificities of five bacterial wax ester synthases. Appl Environ Microbiol 2012, 78:5734-5745.

6. Angerbauer C, Siebenhofer M, Mittelbach M, Guebitz G: Conversion of sewage sludge into lipids by Lipomyces starkeyi for biodiesel production. Bioresour Technol 2008, 99:3051-3056

7. Shi SB, Valle-Rodriguez JO, Siewers V, Nielsen J: Prospects for microbial biodiesel production. Biotechnol J 2011, 6:277-285.

8. Banerjee A, Sharma R, Chisti Y, Banerjee U: Botryococcus braunii: A renewable source of hydrocarbons and other chemicals. Crit Rev Biotechnol 2002, 22:245-279.

9. Gouda MK, Omar SH, Aouad LM: Single cell oil production by Gordonia sp DG using agro-industrial wastes. World J Microbiol Biotechnol 2008, 24:1703-1711.

10. Meng X, Yang J, Xu X, Zhang L, Nie Q, Xian M: Biodiesel production from oleaginous microorganisms. Renew Energy 2009, 34:1-5.

11. Pronk JT, Steensma HY, van Dijken JP: Pyruvate metabolism in Saccharomyces cerevisiae. Yeast 1996, 12:1607-1633.

12. Chen Y, Siewers V, Nielsen J: Profiling of Cytosolic and Peroxisomal AcetylCoA Metabolism in Saccharomyces cerevisiae. PLoS One 2012, 7:e42475.

13. Chen Y, Daviet L, Schalk M, Siewers V, Nielsen J: Establishing a platform cell factory through engineering of yeast acetyl-CoA metabolism. Metab Eng 2013, 15:48-54.

14. Van Hoek P, Van Dijken JP, Pronk JT: Effect of specific growth rate on fermentative capacity of baker's yeast. Appl Environ Microbiol 1998, 64:4226-4233

15. Vemuri GN, Eiteman MA, McEwen JE, Olsson L, Nielsen J: Increasing NADH oxidation reduces overflow metabolism in Saccharomyces cerevisiae. Proc Natl Acad Sci U S A 2007, 104:2402-2407.

16. de Jong-Gubbels $P$, van den Berg MA, Luttick MAH, Steensma HY, van Dijken JP, Pronk JT: Overproduction of acetyl-coenzyme A synthetase isoenzymes in respiring Saccharomyces cerevisiae cells does not reduce acetate production after exposure to glucose excess. FEMS Microbiol Lett 1998, 165:15-20

17. Shiba Y, Paradise EM, Kirby J, Ro D-K, Keasling JD: Engineering of the pyruvate dehydrogenase bypass in Saccharomyces cerevisiae for high-level production of isoprenoids. Metab Eng 2007, 9:160-168.

18. Starai VJ, Gardner JG, Escalante-Semerena JC: Residue Leu-641 of acetyl-CoA synthetase is critical for the acetylation of residue Lys- 609 by the protein acetyltransferase enzyme of Salmonella enterica. J Biol Chem 2005, 280:26200-26205.

19. Tehlivets $\mathrm{O}$, Scheuringer $\mathrm{K}$, Kohlwein SD: Fatty acid synthesis and elongation in yeast. Biochim Biophys Acta 2007, 1771:255-270.

20. Papini M, Nookaew I, Siewers V, Nielsen J: Physiological characterization of recombinant Saccharomyces cerevisiae expressing the Aspergillus nidulans phosphoketolase pathway: validation of activity through C-13-based metabolic flux analysis. Appl Microbiol Biotechnol 2012, 95:1001-1010

21. Panagiotou G, Andersen MR, Grotkjaer T, Regueira TB, Hofmann G, Nielsen J, Olsson L: Systems analysis unfolds the relationship between the phosphoketolase pathway and growth in Aspergillus nidulans. PLoS One 2008, 3:e3847.

22. Kocharin K, Siewers V, Nielsen J: Improved polyhydroxybutyrate production by Saccharomyces cerevisiae through the use of the phosphoketolase pathway. Biotechnol Bioeng 2013, 110:2216-2224.

23. Presecan-Siedel E, Galinier A, Longin R, Deutscher J, Danchin A, Glaser P, Martin-Verstraete I: Catabolite regulation of the pta gene as part of carbon flow pathways in Bacillus subtilis. J Bacteriol 1999, 181:6889-6897.
24. Spector LB: Acetate kinase - a triple-displacement enzyme. Proc Natl Acad Sci USA 1980, 77:2626-2630.

25. Ingram-Smith C, Martin SR, Smith KS: Acetate kinase: not just a bacterial enzyme. Trends Microbiol 2006, 14:249-253.

26. Ratledge C, Holdsworth JE: Properties of a pentulose-5-phosphate phosphoketolase from yeast grown on xylose. Appl Microbiol Biotechnol 1985, 22:217-221.

27. Sonderegger $M$, Schumperli $M$, Sauer U: Metabolic engineering of a phosphoketolase pathway for pentose catabolism in Saccharomyces cerevisiae. Appl Environ Microbiol 2004, 70:2892-2897.

28. Sambrook J, Russel D: Molecular cloning: a laboratory manual. 3rd edition. Cold Spring Harbor, New York: Cold Spring Harbor Laboratory Press; 2001. managing editor: Jan Argentine; ISBN 0-87969-87579-87965.

29. Bertani $\mathrm{G}:$ Studies on lysogenesis.1. The mode of phage liberation by lysogenic Escherichia coli. J Bacteriol 1951, 62:293-300.

30. Chen Y, Bao J, Kim I-K, Siewers V, Nielsen J: Coupled incremental precursor and co-factor supply improves 3-hydroxypropionic acid production in Saccharomyces cerevisiae. Metab Eng 2014, 22:105-109.

31. Partow S, Siewers V, Bjorn S, Nielsen J, Maury J: Characterization of different promoters for designing a new expression vector in Saccharomyces cerevisiae. Yeast 2010, 27:955-964.

32. Chen Y, Partow S, Scalcinati G, Siewers V, Nielsen J: Enhancing the copy number of episomal plasmids in Saccharomyces cerevisiae for improved protein production. FEMS Yeast Res 2012, 12:598-607.

33. Gietz RD, Woods RA: Transformation of yeast by lithium acetate/ single-stranded carrier DNA/polyethylene glycol method. Methods Enzymol 2002, 350:87-96.

34. Khoomrung S, Chumnanpuen P, Jansa-Ard S, Stahlman M, Nookaew I, Boren J, Nielsen J: Rapid Quantification of Yeast Lipid using Microwave-Assisted Total Lipid Extraction and HPLC-CAD. Anal Chem 2013, 85(10):4912-4919.

35. Grabowska D, Chelstowska A: The ALD6 gene product is indispensable for providing NADPH in yeast cells lacking glucose-6-phosphate dehydrogenase activity. World J Biol Chem 2003, 278:13984-13988.

36. Minard Kl, McAlister-Henn L: Sources of NADPH in yeast vary with carbon source. J Biol Chem 2005, 280:39890-39896.

37. van den Berg MA, de Jong-Gubbels P, Kortland CJ, van Dijken JP, Pronk JT, Steensma HY: The two acetyl-coenzyme A synthetases of Saccharomyces cerevisiae differ with respect to kinetic properties and transcriptional regulation. J Biol Chem 1996, 271:28953-28959.

38. Kocharin K, Chen Y, Siewers V, Nielsen J: Engineering of acetyl-CoA metabolism for the improved production of polyhydroxybutyrate in Saccharomyces cerevisiae. AMB Express 2012, 2:52.

39. Zhang Z, MooYoung M, Chisti Y: Plasmid stability in recombinant Saccharomyces cerevisiae. Biotechnol Adv 1996, 14:401-435.

40. Velmurugan S, Mehta S, Uzri D, Jayaram M: Stable propagation of 'selfish' genetic elements. J Biosci 2003, 28:623-636.

41. Dasilva NA, Bailey JE: Influence of plasmid origin and promoter strength in fermentations of recombinant yeast. Biotechnol Bioeng 1991, 37:318-324.

42. Jensen NB, Strucko T, Kildegaard KR, David F, Maury J, Mortensen UH, Forster J, Nielsen J, Borodina I: EasyClone: method for iterative chromosomal integration of multiple genes in Saccharomyces cerevisiae. FEMS Yeast Res 2013, doi: 10.1111/1567-1364.12118.

doi:10.1186/1475-2859-13-39

Cite this article as: de Jong et al:: Improved production of fatty acid ethyl esters in Saccharomyces cerevisiae through up-regulation of the ethanol degradation pathway and expression of the heterologous phosphoketolase pathway. Microbial Cell Factories 2014 13:39. 